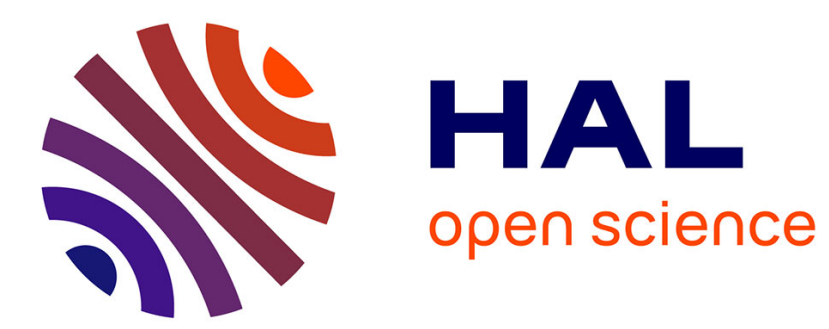

\title{
Fuzzy Piecewise Linear Regression
}

Amory Bisserier, Sylvie Galichet, Reda Boukezzoula

\section{To cite this version:}

Amory Bisserier, Sylvie Galichet, Reda Boukezzoula. Fuzzy Piecewise Linear Regression. 5th IEEE World Congress on Computational Intelligence (WCCI 2008 / FUZZ-IEEE 2008), Jun 2008, HongKong, China. pp. 2089-2094. hal-00447161

\section{HAL Id: hal-00447161 https://hal.science/hal-00447161}

Submitted on 14 Jan 2010

HAL is a multi-disciplinary open access archive for the deposit and dissemination of scientific research documents, whether they are published or not. The documents may come from teaching and research institutions in France or abroad, or from public or private research centers.
L'archive ouverte pluridisciplinaire HAL, est destinée au dépôt et à la diffusion de documents scientifiques de niveau recherche, publiés ou non, émanant des établissements d'enseignement et de recherche français ou étrangers, des laboratoires publics ou privés. 


\title{
Fuzzy piecewise linear regression
}

\author{
A. Bisserier, S. Galichet, R. Boukezzoula
}

\begin{abstract}
Fuzzy regression using possibilistic concepts allows the identification of models from uncertain data sets. However, some limitations still exist about the possible evolution of the output spread with respect to inputs. We present here a modified form of fuzzy linear model whose output can have any kind of output spread tendency. The formulation of the linear program used to identify the model introduces a modified criterion that assesses the model fuzziness independently of the collected data. These concepts are used in a global identification process in charge of building a piecewise model able to represent every kind of output evolution.
\end{abstract}

\section{INTRODUCTION}

Model identification is based on a general principle which consists in determining, among candidate functions, the function of the inputs that best explains the output according to a given criterion. Assuming a particular class of models such as linear functions, splines, rule-based systems, neural networks, ..., the best candidate is determined from the available information, usually a set of observations of the input and output variables. Classical identification techniques assume perfect knowledge of input and output values. It means that the observations are supposed to be both precise (point-valued) and certain. However, there are situations in which this assumption is not realistic, especially when the information about the output value is obtained through measuring devices with limited precision. In the framework of fuzzy modeling in which it is possible to handle imprecise representations using fuzzy set theory as proposed by Zadeh [15], the assumption of perfect data becomes even paradoxical. Nevertheless, most fuzzy model identification techniques used in practice, especially in fuzzy control, still consider crisp data. In this context, the main objective of the paper is to revisit some theoretical works about fuzzy regression techniques [5] and to propose some slight improvements for making their practical use in fuzzy model identification easier.

According to [5], fuzzy regression techniques can be classified into two distinct areas. The first, proposed by Diamond [4], is an adaptation of the classical least squares method and the second, called possibilistic regression, reduces the prob-

The authors are from the Laboratoire d'Informatique, Systèmes, Traitement de l'Information et de la Connaissance (LISTIC), Université de Savoie BP 8043974941 Annecy le vieux, Cedex France

E.mail : amory.bisserier@univ-savoie.fr lem of finding fuzzy coefficients of a regression model to one of mathematical programming. The latter, on which we will focus here, was introduced by Tanaka, Uejima and Asai [12] in a linear context. Several improvements on the original method can be found in the literature. For example, Tanaka, Hayashi and Watada [10] propose different expressions of the criterion to be optimized and different formulations of the constraints to be satisfied for possibility and necessity estimation models. Still in a linear context, Tanaka and Ishibuchi [11] extend their approach for dealing with interactive fuzzy parameters. Furthermore, the complete specification of regression problems highly depends on the nature of input-output data [5]. Some works are thus devoted to crisp input crisp output data [9] while others [8] consider fuzzy input fuzzy output data. Most commonly, a mixed approach (crisp input - fuzzy output) is chosen [12]. That is the formalism we adopt here in a linear context with the idea of keeping a simple model, possibly invertible ([2], [3]).

The expressiveness of linear regression models with fuzzy triangular parameters is limited. One weakness is the fact that the fuzziness of the model output varies in the same way than the absolute value of the inputs. It follows that it is impossible to have a decreasing (resp. increasing) spread of the model output for positive (resp. negative) inputs. This restriction is acceptable in a measurement context where it is usual to express percentage relative errors. However, when fuzziness is considered as an intrinsic characteristic of the system to be modeled, the assumption that the higher the input, the higher the fuzziness attached to the model output, is open to criticism. Finally, as classical fuzzy regression models are not able to represent any tendency of output spread, they become more imprecise than necessary in some situations. As a consequence, in piecewise fuzzy regression problems, in which collected data can have any kind of spread tendency, actual identification methods are clearly insufficient. For example, $\mathrm{Yu}$, Tzeng and $\mathrm{Li}$ [13],[14] propose a piecewise model in which each fuzzy linear piece is constructed according to its difference with the previous piece but the above-mentioned problem is not tackled. Furthermore, from a computational point of view, the choice of a differential model form is not very convenient.

According to above-discussed points, the originality of the presented work is triple:

1) A modified form of fuzzy linear model is proposed so that any kind of spread tendency can be represented. 
2) The formulation of the linear program used to identify the model introduces a modified criterion that assesses the model fuzziness independently of the collected data.

3) These concepts are used in a global identification process in charge of building a piecewise model able to explain every kind of output evolution.

This paper is organized as follows. Section II presents the proposed fuzzy linear model and then addresses its identification. Section III presents the global identification process of a piecewise model. Applications on several examples are shown in Section IV. Then, some discussion about the model validity is developed in Section V. Finally, conclusions and perspectives are presented in Section VI.

\section{IDENTIFICATION OF A MODEL ON AN INTERVAL}

\section{A. Notations and concepts}

We consider symmetrical triangular fuzzy numbers whose notation is $(m, r)$ with $m$ the modal value and $r$ the radius. A representation of this kind of fuzzy numbers is shown in Fig. 1. Let us also introduce the notion of $\alpha$-cut, with $\alpha$ a cut level between 0 and 1 . The $\alpha$-cut of the fuzzy number $A$ is the interval $[\mathrm{A}]_{\alpha}$ composed of elements whose membership in $A$ is greater or equal to $\alpha$, i.e.:

$$
\begin{gathered}
{[A]_{\alpha}=\left\{x \mid \mu_{A}(x) \geq \alpha\right\}} \\
=[m-(1-\alpha) r, m+(1-\alpha) r]
\end{gathered}
$$

The $\alpha$-cut of $A$ at $\alpha=0$ is the support of the fuzzy number, noted $[A]_{0}=\left[A^{-}, A^{+}\right]$(see Fig. 1.).

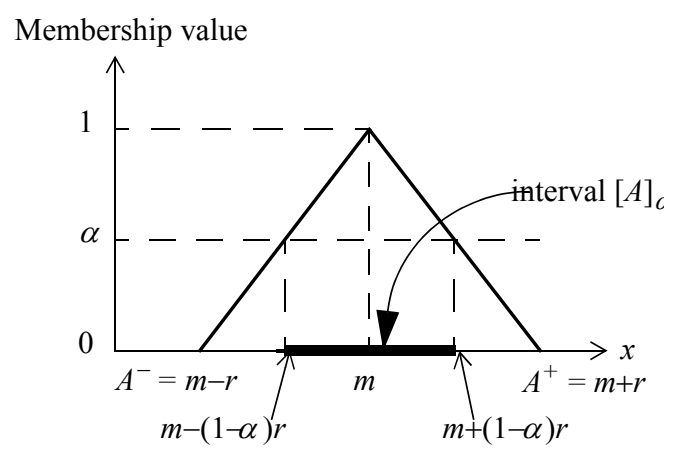

Fig. 1. Representation of a fuzzy number with an $\alpha$-cut.

\section{B. Linear fuzzy model representation}

The objective of this part is to propose a convenient form for a fuzzy linear model. For the sake of simplicity but without lack of generality, we consider here a single input model.

A classical fuzzy linear model with a single input is defined on its domain $D=\left[x_{\min }, x_{\max }\right]$ by:

$$
Y(x)=A_{0} \oplus A_{1} x
$$

where $x$ is the input variable, $x \in D, Y(x)$ the corresponding output, and $A_{0}=\left(m_{A_{0}}, r_{A_{0}}\right)$ and $A_{1}=\left(m_{A_{1}}, r_{A_{1}}\right)$ the two fuzzy coefficients. The operator $\oplus$ represents the sum of two fuzzy numbers, whose result is also a fuzzy number, defined by its modal value and its radius.

As $A_{0}$ and $A_{1}$ are symmetrical triangular fuzzy numbers, and $x$ a crisp input, $Y(x)$ is also a symmetrical triangular fuzzy number. So, its modal value and spread, i.e. $m_{Y(x)}$ and $r_{Y(x)}$ are given by:

$$
\begin{aligned}
& m_{Y(x)}=m_{A_{0}}+m_{A_{1}} x \\
& r_{Y(x)}=r_{A_{0}}+r_{A_{1}}|x|
\end{aligned}
$$

It is obvious from (3) that the variation of $m_{Y(x)}$ with respect to $x$ only depends on $m_{A_{1}}$. So, by choosing the sign of $m_{A_{1}}$, it is possible to represent either increasing or decreasing $m_{Y(x)}$ with respect to $x \in D$. On the contrary, from (4), it appears that the variation of $r_{Y(x)}$ with respect to $x$ only depends on the sign of $x$ since $r_{A_{1}}$ is positive. It follows that when $x$ is positive, $r_{Y(x)}$ is increasing, whereas when $x$ is negative, $r_{Y(x)}$ is decreasing. So, in model (2), it is possible to have any kind of variation of the output modal value with an appropriate sign of $m_{A_{1}}$, but the variation of the output radius is limited by the sign of the input $x$.

Consequently, if we want to obtain a regression model whose output can have any kind of radius variation for any sign of $x$, it is necessary to slightly modify the fuzzy model variable to be able to control its sign. With the idea of keeping a linear behavior, we propose to shift the original input $x$ so as to obtain the desired sign for the shifted variable. So, by defining $(x-$ shift $)$ as the new input variable of the fuzzy model, it becomes possible to control the radius variation by tuning the value of shift.

Thus, we now consider a fuzzy linear model defined on its domain $D=\left[x_{\text {min }}, x_{\text {max }}\right]$ by:

$$
Y(x)=A_{0} \oplus A_{1}(x-\text { shift })
$$

The output modal value and radius are given by:

$$
\begin{aligned}
m_{Y(x)} & =m_{A_{0}}+m_{A_{1}}(x-s h i f t) \\
r_{Y(x)} & =r_{A_{0}}+r_{A_{1}} \mid x-\text { shift } \mid
\end{aligned}
$$

The output modal value variation with respect to $x$ only depends on the sign of $m_{A_{1}}$. So, increasing and decreasing tendencies can be represented. Concerning the variation of $r_{Y(x)}$ with respect to $x$, two cases are possible depending on the sign of $x$-shift. When an increasing $r_{Y(x)}$ is desired, it is necessary that $x-$ shift $\geq 0$, i.e. $x \geq$ shift. As $x \in\left[x_{\min }, x_{\max }\right]$, it is required that shift $\leq x_{\min }$. On the contrary, for a decreasing $r_{Y(x)}$, it is necessary that $x-$ shift $\leq 0$, i.e. $x \leq$ shift . This constraint has to be satisfied for any $x \in D$, which means that the shift value has to be chosen so that shift $\geq x_{\max }$.

In the remaining, a shift convenient value is chosen. More precisely, the bound of the domain $D$ that satisfies the constraint associated to the tendency desired for $r_{Y(x)}$ is selected. In other words, shift $=x_{\min }$ for a model whose output has an increasing radius with respect to $x$ and shift $=x_{\max }$ for a decreasing radius (see TABLE I). It amounts to positioning the zero of the shifted model either at $x_{\min }$ or at $x_{\max }$. By doing so, the output $Y(x)$ of the fuzzy model (5) is equal to $A_{0}$ at one 
bound of the domain $D$.

TABLE I

THE TWO MODELS

\begin{tabular}{|c|c|c|}
\hline $\begin{array}{c}\text { output radius varia- } \\
\text { tion }\end{array}$ & 7 & $\mathbf{V}$ \\
\hline model used & $A_{0} \oplus A_{1}\left(x-x_{\text {min }}\right)$ & $A_{0} \oplus A_{1}\left(x-x_{\text {max }}\right)$ \\
\hline
\end{tabular}

The shifted model (5) can be characterized by its global fuzziness, possibly assessed as the area covered by the fuzzy output when the input varies on its domain $\left[x_{\text {min }}, x_{\text {max }}\right]$. By denoting $w=(x-s h i f t)$ the shifted model input and $\left[w_{\text {min }}\right.$, $\left.w_{\text {max }}\right]$ its variation domain, the global fuzziness of the model is given by:

$$
2\left(w_{\max }-w_{\min }\right) r_{A_{0}}+\left(\left|w_{\text {max }}^{2}-w_{\text {min }}^{2}\right|\right) r_{A_{1}} .
$$

The model identification procedure described in the next section is based on the following principle: considering two acceptable models, the one with the minimum fuzziness, i.e. the less imprecise is preferred.

\section{Fuzzy model identification}

The identification of a shifted fuzzy model (5) on a given domain $D=\left[x_{\text {min }}, x_{\text {max }}\right]$ amounts to determine the shift value and the two fuzzy coefficients $A_{0}$ and $A_{1}$. The latter being symmetrical triangular fuzzy numbers, it consists in finding their modal values $m_{A_{0}}$ and $m_{A_{1}}$ and their spreads $r_{A_{0}}$ and $r_{A_{1}}$. A set of $M$ data is available for the identification. In all input/ output observations $\left(x_{j}, Y_{j}\right), j=1, \ldots, M$, the input $x_{j}$ is a crisp value in $D$ and the corresponding output $Y_{j}$ is a fuzzy number, noted $\left(y_{j}, e_{j}\right)$ where $y_{j}$ is the modal value and $e_{j}$ the radius. The $M$ data are sorted in increasing order of $x_{j}$.

The first step of the identification concerns the choice of the shift value according to the output radius tendency (see TABLE I). The most appropriate tendency is determined from observed data, comparing the initial output radius $r_{\text {init }}$ attached to minimal inputs with the final output radius $r_{\text {fin }}$ attached to maximal inputs. If $r_{\text {init }}<r_{\text {fin }}$, an increasing tendency is chosen, otherwise a decreasing tendency is preferred. The corresponding shift value is determined from TABLE I. The $r_{\text {init }}$ and $r_{\text {fin }}$ values are estimated by computing mean values from $k$ data, that is $r_{\text {init }}=\operatorname{mean}\left(e_{1}, e_{2}, \ldots, e_{k}\right)$ and $r_{\text {fin }}=$ $\operatorname{mean}\left(e_{M-k+1}, \ldots, e_{M-1}, e_{M}\right)$.

The next step of the identification concerns the optimization of the fuzzy coefficients $A_{0}$ and $A_{1}$. A fuzzy regression approach based on possibilistic concepts as introduced by Tana$\mathrm{ka}$ [12] is used. It consists in solving a linear programming problem, with a criterion to be optimized and constraints to be satisfied. Hereinafter, the shifted variable $w_{j}=\left(x_{j}-s h i f t\right)$ is used in order to simplify the criterion expression and the constraint writing. Note that $w_{j}$ is either positive or negative, depending on the shift value.
If several kinds of constraints can be considered [10], we want hereinafter the predicted intervals (resulting of the $\alpha$-cut of fuzzy numbers) to fully cover the observed ones. Considering the support of observed fuzzy numbers, i.e. $\alpha=0$, the whole fuzziness of data is taken into account in the model identification. So, at $\alpha=0$, with $\hat{Y}_{j}=\left[\hat{Y}_{j}^{-}, \hat{Y}_{j}^{+}\right]$the $j^{\text {th }}$ predicted support, the following constraint is obtained:

$$
\left[y_{j}-e_{j}, y_{j}+e_{j}\right] \subseteq\left[\hat{Y}_{j}^{-}, \hat{Y}_{j}^{+}\right]
$$

otherwise expressed as:

$$
\begin{gathered}
m_{A_{0}}+m_{A_{1}} w_{j}+r_{A_{0}}+r_{A_{1}}\left|w_{j}\right| \geq y_{j}+e_{j} \\
m_{A_{0}}+m_{A_{1}} w_{j}-\left(r_{A_{0}}+r_{A_{1}}\left|w_{j}\right|\right) \leq y_{j}-e_{j}
\end{gathered} .
$$

The choice of the criterion to be minimized is also an important issue. The first one introduced by Tanaka, Uejima and Asai [12] is the sum of the spreads of the model coefficients, which leads to the following optimization problem:

$$
m_{A_{0}}, m_{A_{1}}, r_{A_{0}}, r_{A_{1}} r_{A_{0}}+r_{A_{1}}
$$

Another approach, consisting in minimizing the sum of the spreads of the predicted intervals, also exists:

$$
\min _{m_{A_{0}}, m_{A_{1}}, r_{A_{0}}, r_{A_{1}}} M r_{A_{0}}+r_{A_{1}} \sum_{j=1}^{M}\left|w_{j}\right|
$$

It is well established in the literature that the criterion (12) is more relevant than the criterion (11). Indeed, the use of the criterion (12) improves the predictivity of the identified model. However, as the criterion (12) is only based on the available data, its minimization does not guarantee that the identified model has the least global fuzziness (8) that could be achieved on the whole domain $D$. If the identified model is to be used on the whole domain $D$, it may be more judicious to prefer a model with a lower global fuzziness, i.e. a less imprecise model. In this context, the global fuzziness (8) becomes the criterion to be minimized as proposed in [1]. Actually, the global fuzziness of a model is an intrinsic characteristic of the latter, that should be assessed independently of the identification data, already used to express the constraints of the linear program.

According to this criterion choice, the following optimization problem is obtained:

$$
\min _{m_{A_{0}}, m_{A_{1}}, r_{A_{0}}, r_{A_{1}}} 2\left(w_{\text {max }}-w_{\text {min }}\right) r_{A_{0}}+\left(\left|w_{\text {max }}^{2}-w_{\text {min }}^{2}\right|\right) r_{A_{1}}
$$

In order to compare problems (12) and (13), both criteria are normalized. The normalization of (12) leads to:

$$
\min _{m_{A_{0}}, m_{A_{1}}, r_{A_{0}}, r_{A_{1}}} r_{A_{0}}+r_{A_{1}} \bar{w}
$$

where $\bar{w}$ is the average of $w_{j}, j=1, \ldots, M$, while the normalization of (13) results in: 


$$
\min _{m_{A_{0}}, m_{A_{1}}, r_{A_{0}}, r_{A_{1}}} r_{A_{0}}+r_{A_{1}} w_{m}
$$

where $w_{m}$ is the middle point of the interval $\left[w_{\min }, w_{\max }\right]$ :

$$
w_{m}=\frac{\left(w_{\max }+w_{\min }\right)}{2} .
$$

So, if the identification data are uniformly distributed on the domain $D$, the two criteria are strictly equivalent. If it is not the case, solving problem (13) may lead to a model less imprecise than solving problem (12). This approach can be interesting especially when $M$ is small or when the domain $D$ is bounded and clearly specified.

To sum up, the identification on $D=\left[x_{\min }, x_{\max }\right]$ of a model $\hat{Y}(x)=A_{0} \oplus A_{1}(x-$ shift $)$ using a set of $M$ observed data, is dealt with the following Identification function.

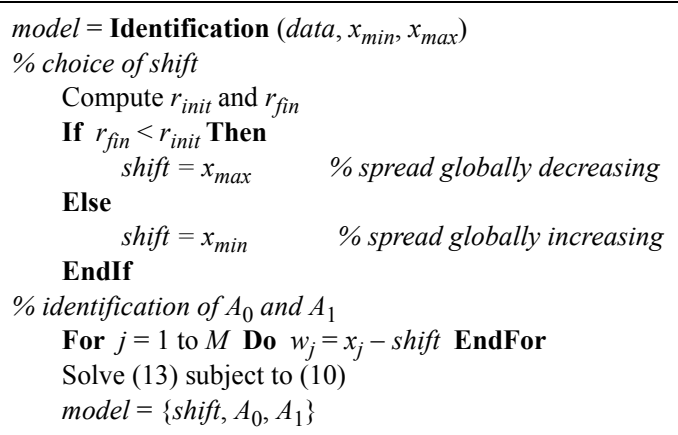

\section{PIECEWISE LINEAR MODEL IDENTIFICATION}

The fuzzy piecewise identification method is divided into two tasks: structure identification and parameter estimation. The structure identification consists in determining the number of submodels and their intervals of definition according to a segmentation method. In this context, several techniques have been developed to deal with this problem. Of course, any segmentation method can be applied. However, as already pointed out in [7], the quality of the model identification depends on the applied segmentation procedure. So, when a segmentation method is available, the identification problem is reduced to a parameters estimation one. That is the approach developed in this paper where the principle proposed by Keogh, Chu, Hart and Pazzani for segmenting time series [7] is used.

The proposed identification method is used to identify a piecewise fuzzy linear model of the form (17):

$$
\hat{Y}(x)=\sum_{k=1}^{N}\left[A_{k 0} \oplus A_{k 1}\left(x-\text { shift }_{k}\right)\right] 1_{\left[x_{\min }^{k}, x_{\max }^{k}\right]}
$$

where $\operatorname{shift}_{k} \in\left\{x_{\text {min }}^{k}, x_{\text {max }}^{k}\right\}, N$ is the number of submodels which compose the global model, $\sum^{\oplus}$ represents the sum of fuzzy numbers. The function $1 c^{k} x^{k}$ is equal to 1 on $\left[x_{\min }^{k}, x_{\max }^{k}\right]$ and to 0 anywhere else. $\left[x_{\min }, x_{\max }^{k}\right]$

In the identification method implementation some considerations must be taken into account. At the beginning of the process, it is necessary to find a good segmentation of the data set, i.e. to determine in which intervals to identify the different submodels. A changeover between two submodels is characterized either by an abrupt change in output modal values or by an abrupt change in output radius. So, in the segmentation procedure, both changes have thus to be considered. So, in order to get the different $\left[x_{\text {min }}^{k}, x_{\text {max }}^{k}\right]$, $k=1, \ldots, N$, the following strategy is applied:

- segmentation according to $y_{j}$

- on each interval got, segmentation according to $e_{j}$.

In this context, the Identification function is applied on each interval given by any segmentation process in order to determine the best model according to the global fuzziness criterion (8).

The advantage of the proposed method with regard to the one developed by Yu, Tzeng and Li [14] resides in the two following points:

- from a structural point of view, according to the suitable shifts determination, any kind of output spread tendencies can be represented.

- from a practical application point of view, the obtained piecewise model is not differential (the submodels can be identified and used independently).

Let's have a few words about the continuity of the global model. Actually, the proposed identification procedure do not require the model continuity because looking for a continuous model may strongly increase the model fuzziness. Indeed, considering two consecutive segments, the more imprecise model induces an additional constraint on the other model when continuity is imposed. Moreover, there is no negative consequence on the use of the model, in case of inversion for example, if an 0 -order holder is implemented at the change points.

\section{NUMERICAL EXAMPLES}

First, we apply our identification method to the example proposed by $\mathrm{Yu}$, Tzeng and $\mathrm{Li}$ [14].

The model we get is the following (18):

$$
\begin{aligned}
\hat{Y}(x) & =((11.5,1.5)+(-1,0.333)(x-6)) 1_{[3,6]} \\
& +((12.5,2.5)+(0.833,0.167)(x-6)) 1_{[6,15]} \\
& +((17.5,1.5)+(-1.333,0.337)(x-18)) 1_{[15,18]}
\end{aligned}
$$

Its global fuzziness (8) is 82.5 , whereas the Yu model [14] has a global fuzziness equal to 99 . The model (18) is clearly less fuzzy and so can be considered as better.

In order to confirm the performance of the obtained models, other criteria have to be considered. In this context, the sum of the spreads of the predicted intervals (see (12)), named Sum, and the Diamond distance [4] between the observed and the predicted fuzzy numbers, named Dist. are used. For this example, the obtained results are summarized in Table II. 
TABLE II

TWO PERFORMANCE INDICATORS

\begin{tabular}{|c|c|c|}
\hline model & Sum & Dist \\
\hline Yu model [14] & 20 & 14.5 \\
\hline Our model (18) & 15 & 7 \\
\hline
\end{tabular}

According to Table II, it can be stated that the obtained model presents a better fitting of the data, i.e., both Sum and Dist are lower.

Then, we apply our identification method to the example proposed by Tanaka and Ishibuchi ([11], example 2).

The model we get is the following (19) (see Fig. 2.):

$$
\begin{aligned}
\hat{Y}(x)= & ((10,1)+(0.5,0.5)(x-11)) 1_{[5,11]} \\
& +((10,1)+(0.5,1.33)(x-11)) 1_{[11,17]}
\end{aligned}
$$

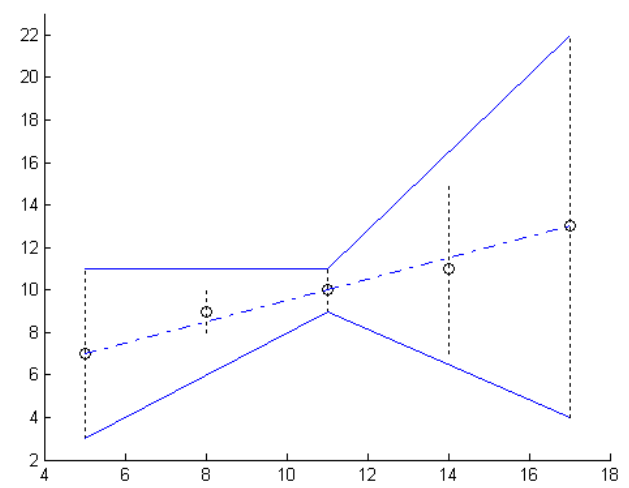

Fig. 2. Identified model on the example of Tanaka and Ishibuchi

Whereas Tanaka and Ishibuchi [11] identify a model with interactive parameters, thus introducing quadratic membership functions, we handle the spread variations in a piecewise lin- ear context with triangular fuzzy coefficients.

Finally, we apply our identification technique on synthetic time serie data, with several cases of modal value and spread tendencies. Used data are too numerous to be presented here, so we only explain how the data set is generated (see Table III).

On each interval, the input $x$ is increasing with an increment of 1 . For each linear submodel, we set a variation for the modal value and another for the spread, then we add a random noise. Note that for the first two intervals, spread is globally constant, and so we only add a noise between 15 and 25 for the first one, and 5 and 10 for the other one, in order to get a change in spread level. The model identified from the data is presented in Table III and shown in Fig. 3.

TABLE III

DATA CONSIDERED AND IDENTIFIED MODEL

\begin{tabular}{|c|c|c|c|c|c||}
\hline interval & $\begin{array}{c}\text { modal } \\
\text { tendency }\end{array}$ & spread & $A_{0}$ & $A_{1}$ & shift \\
\hline$[1,25]$ & 20 & $\begin{array}{c}\text { random } \\
{[15,25]}\end{array}$ & $(24.3,25.6)$ & $(-0.1,0)$ & 1 \\
\hline$[26,50]$ & 20 & $\begin{array}{c}\text { random } \\
{[5,10]}\end{array}$ & $(23.9,9.2)$ & $(-0.1,0.1)$ & 26 \\
\hline$[51,75]$ & $1.5 x+1$ & $3 / 2 x$ & $(27.6,4.9)$ & $(1.4,1.5)$ & 51 \\
\hline$[76,100]$ & $1.5 x+1$ & $-3 / 2 x$ & $(102.9,5.6)$ & $(1.4,1.3)$ & 100 \\
\hline$[101,125]$ & 100 & $2 x$ & $(101.6,7.9)$ & $(-0.1,2.1)$ & 101 \\
\hline$[126,150]$ & 100 & $-x$ & $(103.5,3.9)$ & $(0.1,1.9)$ & 150 \\
\hline$[151,175]$ & $-x$ & $x$ & $(104.3,7.8)$ & $(-0.9,0.9)$ & 151 \\
\hline$[176,200]$ & $-x$ & $-x$ & $(51.4,7.8)$ & $(-1.1,0.8)$ & 200 \\
\hline$[201,225]$ & $-x$ & $1.5 x$ & $(51.8,9.4)$ & $(-0.9,1.5)$ & 201 \\
\hline
\end{tabular}

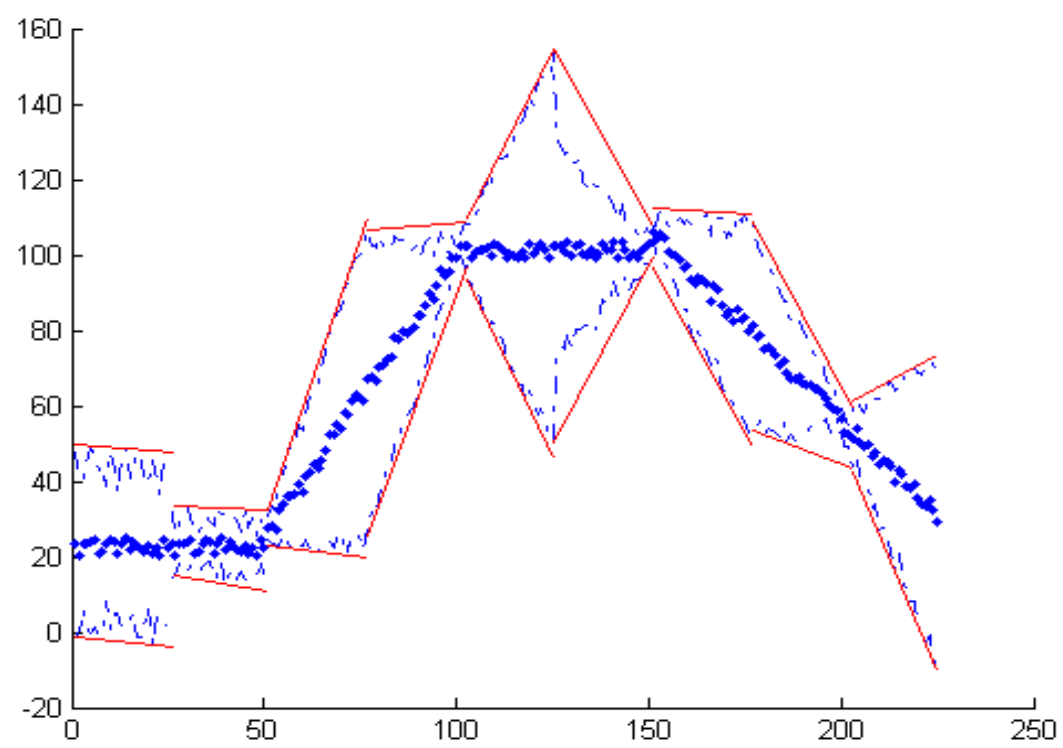

Fig. 3. Identified model on a time serie data set 
We can see that we get a good identification of a piecewise model on the data set. With the new kind of models with shift, we get a well suited representation of the observed data on each interval, and this, for any output spread tendency.

\section{ABOUT THE VALIDITY OF THE MODEL}

The identification method we propose here is based on possibilistic concepts. The main aspect of this kind of method is the use of a linear program. In the literature, several kinds of criteria or constraints are proposed in order to improve the identification of the model. However, all these methods have a similar aspect, i.e. the use of $\alpha$-cuts in order to finally consider intervals in the optimization procedure. Choosing some $\alpha$ level, a modal value and a radius are determined for each coefficient of the model with the necessity of having inclusion of the $\alpha$-cut of the observed output $\left[Y_{j}\right]_{\alpha}$ in the predicted one $\left[\hat{Y}_{j}\right]_{\alpha}$ (see Fig. 4.). Then, the fuzzy model output is reconstructed, for each value of $\alpha$ between 0 and 1 . So, it is possible for any value of the input to determine the corresponding fuzzy output.

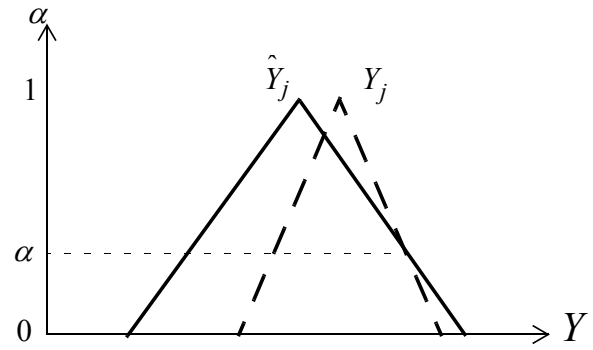

Fig. 4. Observed and predicted fuzzy numbers for a given input $x_{j}$

Two points appears clearly in Fig. 4. Whatever the chosen $\alpha$ level in the optimization problem, for any $\alpha^{\prime} \leq \alpha$, the inclusion of the observed intervals in the predicted ones is respected. However, it is not the case anymore for any $\alpha^{\prime \prime}>\alpha$. Consequently, even if the inclusion is respected at a given $\alpha$ level, it is not the case for the whole fuzzy number after reconstruction.

An idea that can be exploited is to identify the model at $\alpha=1$. However, it is well known from the literature that the optimization problem has no solution when the observed modal values are not strictly lined up. Moreover, the higher the $\alpha$ considered for the identification, the wider the support of the predicted fuzzy number. That's why we use in the work presented here a linear program at $\alpha=0$, in order to get the most relevant model taking in care the whole fuzziness of the observed data, i.e. the model with the less wide support.

However, in our opinion the reconstruction of a fuzzy set from an interval-based identification is not pertinent in a fuzzy point of view. However, as this approach is classically used in the literature, we adopt the same formalism in order to position our technique and results with respect to other existing methods. Knowing this important limit, we work at present on the identification of another form of fuzzy models whose output could contain all fuzzy observed data.

\section{CONCLUSION}

With the proposed form of shifted models, it becomes possible to represent output spreads either increasing or decreasing with respect to inputs. Identifying such models leads to models whose fuzziness is possibly lower than usually. Moreover, in the context of piecewise modeling, the double segmentation, one for the modal values of the observed outputs and another for their spreads, contributes towards reducing the global fuzziness of the model.

Further works concern the extension of the proposed approach to multi-input problems. Actually, the regression algorithm is easily adaptable, but the way the multidimensional input space should be segmented remains a crucial problem. Another point to be studied is the generalization of the identification procedure to fuzzy inputs, in order to manage several cases of uncertainty on collected data.

\section{REFERENCES}

[1] A. Bisserier, S. Galichet, R. Boukezzoula, Une vision de la régression linéaire floue au travers de l'arithmétique des intervalles, Rencontres francophones sur la Logique Floue et ses Applications (LFA'2007), Nîmes, France, 2007, pp. 57-64.

[2] R. Boukezzoula, S. Galichet, L. Foulloy, Nonlinear Internal Model Control: Application of Inverse Model Based Fuzzy Control, IEEE Transactions on Fuzzy Systems, Vol. 11, No. 6, 2003, pp. 814-829.

[3] R. Boukezzoula, L. Foulloy, S. Galichet, Inverse Controller Design for Interval Fuzzy Systems, IEEE Transactions On Fuzzy Systems, Vol. 14, No. 1, February, 2006, pp. 111-124.

[4] P. Diamond, Fuzzy Least Squares, Information Sciences 46: 141-157, 1988.

[5] P. Diamond, H. Tanaka, Fuzzy regression analysis, Fuzzy sets in decision analysis, operations research and statistics, Kluwer Academic Publishers, Norwell, MA, 1999.

[6] D. Dubois, H. Prade, Fuzzy sets and systems: theory and applications, Academic Press, New York, 1980.

[7] E. Keogh, S. Chu, D. Hart, M. Pazzani, "An Online Algorithm for Segmenting Time Series" in First IEEE International Conference on Data Mining (ICDM'01), p. 289-297, 2001.

[8] M. Sakawa, H. Yano, Mutiobjective fuzzy linear regression analysis for fuzzy input-ouput data, Fuzzy Sets and Systems 47: 173-181, 1992

[9] D.A. Savic, W. Pedrycz, Evaluation of fuzzy linear regression models, Fuzzy Sets and Systems 39: 51-63, 1991.

[10] H.Tanaka, I. Hayashi, J. Watada, Possibilistic linear regression analysis for fuzzy data, European Journal of Operational Research 40: 389-396, 1989.

[11] H. Tanaka, H. Ishibuchi, Identification of possibilistic linear systems by quadratic membership functions of fuzzy parameters, Fuzzy Sets and Systems 41: 145-160, 1991.

[12] H.Tanaka, S. Uejima, K. Asai, Linear regression analysis with fuzzy model, IEEE, Systems, Trans. Systems Man Cybernet. SMC-2: 903-907, 1982.

[13] J.R. Yu, G.H. Tzeng, H.L. Li, A general piecewise necessity regression analysis based on linear programming, Fuzzy Sets and Systems 105: 429-436, 1999.

[14] J.R. Yu, G.H. Tzeng, H.L. Li, General fuzzy piecewise regression analysis with automatic change-point detection, Fuzzy Sets and Systems 119: 247-257, 2001.

[15] L.A. Zadeh, Fuzzy Sets, Information and Control 8: 338-353, 1965. 